\title{
Investigación Básica
}

\section{Identificación y cuantificación del arsénico unido a las proteínas de tejidos cardiovasculares de pacientes sometidos a cirugía de revascularización coronaria}

\author{
Isabel Pizarro ${ }^{1}$, Domingo Román ${ }^{1}$, Carlos Solar ${ }^{2}$, Carmen Cámara ${ }^{3}$, María Antonia Palacios ${ }^{3}$, María \\ Milagros Gómez ${ }^{3}$.
}

1 Laboratorio de Química Bioinorgánica y Analítica Ambiental.
Departamento de Química. Facultad de Ciencias Básicas. Universidad de Antofagasta - Chile
2 Unidad de Cirugía Cardiovascular. Clinica Antofagasta - Chile
3 Departamento de Química Analítica. Facultad de Ciencias Químicas. Universidad Complutense de Madrid España

Introducción: Los efectos de la intoxicación con Arsénico (As) como enfermedades cardiovasculares (CV), pigmentaciones y oclusiones arteriales coronarias están asociados con la ingestión de As inorgánico a través del agua de bebida y a exposiciones ambientales. La unión del As (III) a proteínas y la metilación del As podría ser una primera etapa en el mecanismo de detoxificación.

Objetivo: Evaluar la unión de As a proteínas en aurícula derecha y vena safena (VS) en sujetos expuestos de la Región de Antofagasta.

Métodos: Se estudió la asociación As-proteína en el citosol de AD y VS de 6 pacientes con enfermedad coronaria grave de la Región de Antofagasta. Para el fraccionamiento del citosol se utilizaron columnas de exclusión molecular de tres diferentes rangos de masas. El perfil del As se detectó por Espectrometría de Masas Inductivamente Acoplado (ICP-MS) y por Espectroscopía Ultra Violeta - Visible de las fracciones moleculares (enlaces As- tiolatos de proteínas).
Resultados: En todos los casos el As estuvo ampliamente distribuido en todo el intervalo de fracciones para AUD y VS. Los porcentajes de As colectado en las fracciones de las diferentes columnas usadas fueron 10 , 25 y $50 \%$. En la especiación de As en el citosol, por Cromatografía Líquida de Alta Resolución acoplada a la Espectrometría de Masas (IC-HPLC-ICP-MS), solamente se encontró As(III) y As(V) con una distribución Gaussiana para ambas especies, siendo la relación $\mathrm{As}(\mathrm{III}) / \mathrm{As}(\mathrm{V})$ constante para AUD y VS.

Conclusión: En los tejidos CV existe asociación As - proteína lo cual podría implicar que el As está unido a biocompuestos de diferente peso molecular a través de grupos sulfhidrilos vecinales. Es probable que el As en AUD y VS se una a fracciones proteicas de masa molecular superior a $80 \mathrm{kDA}$ y a subunidades de la estructura cuaternaria de la proteína nativa.

Palabras Claves: Arsénico, unión a proteínas, tejidos cardiovasculares. 


\section{Identification and quantification of protein-linked arsenic in cardiovascular tissues from patients submitted to coronary revascularization}

Background: The effects of arsenic (As) toxicity - cardiovascular disease, pigmentation, coronary artery occlusion- come from ingestion of contaminated drinking water and environmental exposure. Protein linkage or As(III) and As methylation may be a first step in detoxification. The aim of this study is to evaluate protein linkage of As in the right atrium (RA) and saphenous vein (SV) of As exposed subjects from Antofagasta, Chile

Method: As-protein linkage was studied in the cytosol of $\mathrm{AD}$ and $\mathrm{SV}$ obtained from 6 patients operated on for coronary artery disease. Molecular exclusion columns of 3 different mass ranges were used to obtain the cytosol fraction. As species were detected by induction coupled mass spectrometry and visible ultraviolet

\section{Introducción}

Los efectos clínicos típicos de la intoxicación con arsénico (As) como la enfermedad vascular periférica ${ }^{1}$, pigmentaciones anormales y enfermedad coronaria, están asociadas con la ingestión de As inorgánico a través del agua de bebida y exposiciones ambientales ${ }^{2}$.

La metilación del As inorgánico a monometilarsénico (MMA) o dimetilarsénico (DMA) es un mecanismo de detoxificación muy conocido. Sin embargo, la metilación va precedida de una reducción de arsenato (As (V)) a arsenito (As (III)), especie a partir de la cual se produce la metilación. La unión del As (III) a las proteínas podría ser una primera etapa en el mecanismo de detoxificación. Por lo tanto, antes de que ocurra la metilación, el As puede ser tóxico para el organismo.

La toxicidad del As puede estar relacionada con la degradación de las proteínas o con la limitación de la síntesis de spectrometry (links of As and protein thyolates). Results: As was widely distributed in $\mathrm{AD}$ and $\mathrm{SV}$ in all subjects. As collected in the 3 different columns used were $10 \%, 25 \%$ and $50 \%$. Only As(III) and As(V) were obtained through the method used (IC-HPLCICP-MS); a normal distribution was evident for both As species. The relation As(III)/As(V) was similar in $\mathrm{AD}$ and SV.

Conclusion: A linkage of As and proteins through neighbor sulphidryl groups is present in cardiovascular tissues of exposed subjects. It is likely that As is linked to $>80 \mathrm{kDA}$ protein fractions and to quaternary subunits or the native protein.

Keywords: arsenic, protein lynkage, cardiovascular tissues nuevas proteínas que pueden ser alteradas por As (III). La reactividad del As (III) depende de los enlaces que forma con grupos funcionales como tiolatos, con cisteínas vecinales de enzimas como la piruvato deshidrogenasa y los nitrógenos imidazólicos de los residuos de histidina ${ }^{3}$. Esto permite deducir que la interacción de As con proteínas es esencial para comprender los mecanismos que regulan la química bioinorgánica relacionada con su actividad biológica.

El As (V) interrumpe la fosforlilación oxidativa mediante la sustitución de fosfatos en la formación de ATP $^{4}$, lo que estaría directamente relacionado con su toxicidad.

La unión de As a diversas proteínas citosólicas y procesos de metilación se consideran mecanismos de desintoxicación competitivos ${ }^{5}$ como S-adenosylmetionina y metiltransferasa. Sin embargo, existe evidencia experimental que sugiere que la biometilación, especialmente los meta- 
bolitos metilados que contienen As (III), es un proceso que se activa como una toxina y un carcinógeno ${ }^{6}$.

Se han realizado relativamente escasos estudios de la unión de arsénico a proteínas en los seres humanos ${ }^{5,7}$. No se ha encontrado en la actualidad un modelo que correlacione la toxicidad del arsénico inorgánico con datos clínicos en seres humanos ${ }^{8}$.

En la literatura se mencionan cuatro proteínas unidas a arsénico de 50, 42, 38,5 y 19,5 kDa. Dos de ellas fueron identificadas tentativamente como tubulina $(50 \mathrm{kDa})$ y actina ${ }^{9} 42 \mathrm{kDa}$ ), que son inducidas por As (III) en células humanas linfoblastoides ${ }^{10}$.

En el presente trabajo se evalúa la unión intracelular As-proteína en los tejidos de la AUD y la VS en sujetos expuestos a As que han requerido cirugía de revascularización coronaria.

\section{Métodos}

Muestras: Se analizaron los tejidos de AUD y VS de 6 personas sometidas a cirugía cardíaca en la Clínica Antofagasta, que presentaban alto contenido de As en ambos tejidos ${ }^{1}$. Los pacientes residían al menos cinco años en la Región de Antofagasta - Chile. La preparación de las muestras se realizó en una campana de flujo laminar (Labconco, Purifer clase II), utilizando dispositivos inertes de titanio, mortero de ágata y material quirúrgico de acero inoxidable. Después de la eliminación de residuos, los tejidos fueron enjuagados con agua desionizada, separados como muestras unitarias y almacenados a $-20^{\circ} \mathrm{C}$. La Tabla 1 muestra las características de los pacientes en estudio.

Preparación del citosol: Se preparó el citosol triturando el tejido con solución reguladora Tris de $\mathrm{pH}=7.3$ en pre- sencia de otros agentes tales como el $\mathrm{NaCl}$, que favorecen la extracción de la proteína a la fracción soluble y agentes inhibidores enzimáticos para minimizar la degradación de la proteína. Esta preparación no mantiene intacta a la proteína original, pero permanece la unión del As al resto proteico.

Posteriormente, se realizó el fraccionamiento proteico del citosol y la caracterización de la unión As-proteína en las fracciones proteicas obtenidas .

Fraccionamiento y caracterización de la unión Asproteína: El fraccionamiento de las proteínas presentes en el citosol se realizó por medio de columnas cromatográficas de exclusión por tamaños (Superdex $0.1-7 \mathrm{kDa}$, Phenomenex 1- $300 \mathrm{kDa}$ ) o por columnas de intercambio aniónico de proteínas (MonoQ HR5/5) y empleando Tris como eluyente. Se analizó la proteína total por el método de Bradford ${ }^{11}$, el As total ${ }^{1}$ y la especiación ${ }^{1}$ química de As (III) y As (V).

Se verificó la unión de As a proteínas mediante el acoplamiento en línea del tándem formado por una columna aniónica (MonoQ HR5/5), un detector de radiación ultravioleta (UV), que detecta el paso de la proteína por el sistema y el detector de masas (ICP-MS) que detecta el paso de As que pudiera estar unido a la proteína .

El estudio fue aprobado por el Comité de Bioética de la Universidad de Antofagasta y Clínica Antofagasta, siguiendo el protocolo para donación de muestras por seres humanos.

\section{Resultados}

As Total: En la Tabla 1 se muestran las concentraciones de As total en AUD y VS de los sujetos junto a características clínicas. Las mayores concentraciones de As total

\begin{tabular}{|c|c|c|c|c|c|c|}
\hline \multicolumn{4}{|c|}{ As total $(\mu \mathrm{g} / \mathrm{g}-1)$} & \multicolumn{3}{|c|}{ Información de pacientes en estudio } \\
\hline Muestra & AUD & VS & Edad & Lugar de trabajo & Estigmas de Arsenicismo & Otros \\
\hline S-1 & $3.9 \pm 0.2$ & $2.5 \pm 0.2$ & 37 & Minería (Calama) & $\mathrm{Si}$ & Diabetes, dislipidemia \\
\hline S-2 & $4.5 \pm 0.3$ & $3.2 \pm 0.2$ & 44 & Minería (Chuquicamata) & $\mathrm{Si}$ & Diabetes \\
\hline $\mathrm{S}-3$ & $5.4 \pm 0.4$ & $2.6 \pm 0.3$ & 45 & Minería (Calama) & $\mathrm{Si}$ & - \\
\hline S-4 & $4.8 \pm 0.3$ & $4.7 \pm 0.3$ & 50 & Minería (Antofagasta) & $\mathrm{Si}$ & - \\
\hline S-5 & $4.8 \pm 0.4$ & $5.2 \pm 0.4$ & 53 & Minería (Chuquicamata) & $\mathrm{Si}$ & Diabetes \\
\hline S-6 & $6.1 \pm 0.5$ & $5.1 \pm 0.4$ & 65 & Comerciante (Antofagasta) & $\mathrm{Si}$ & - \\
\hline
\end{tabular}




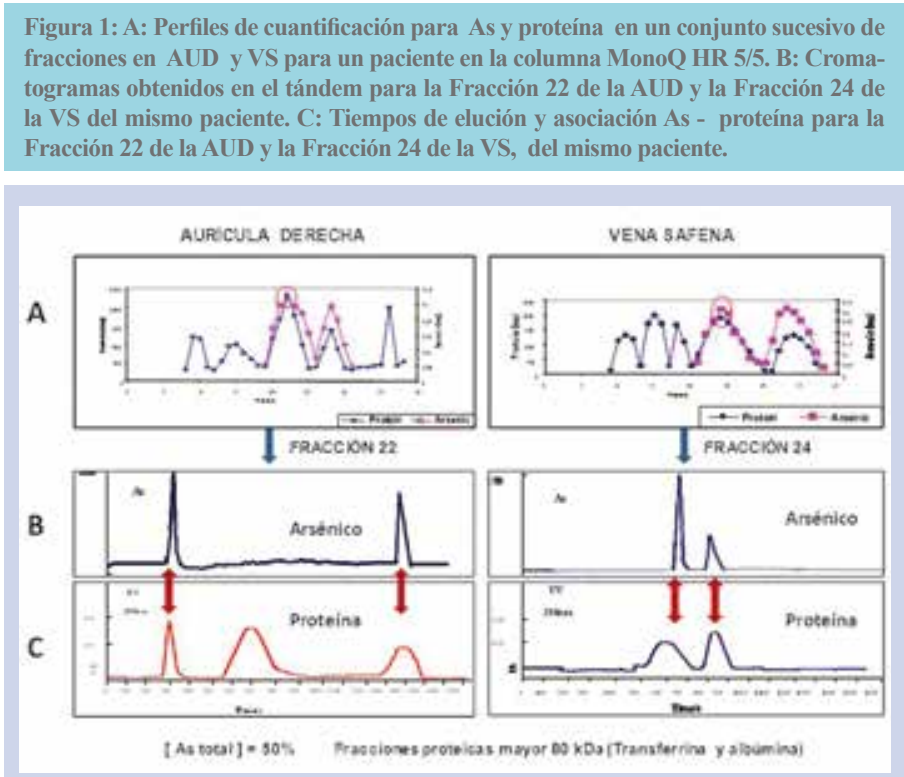

Figura 2. A) Perfiles de cuantificación para As y proteína en un conjunto sucesivo de fracciones en AUD y VS para un paciente en la columna Superdex. B) Perfiles de cuantificación para As y proteína en un conjunto sucesivo de fracciones en AUD y VS para un paciente en la columna Phenomenex.

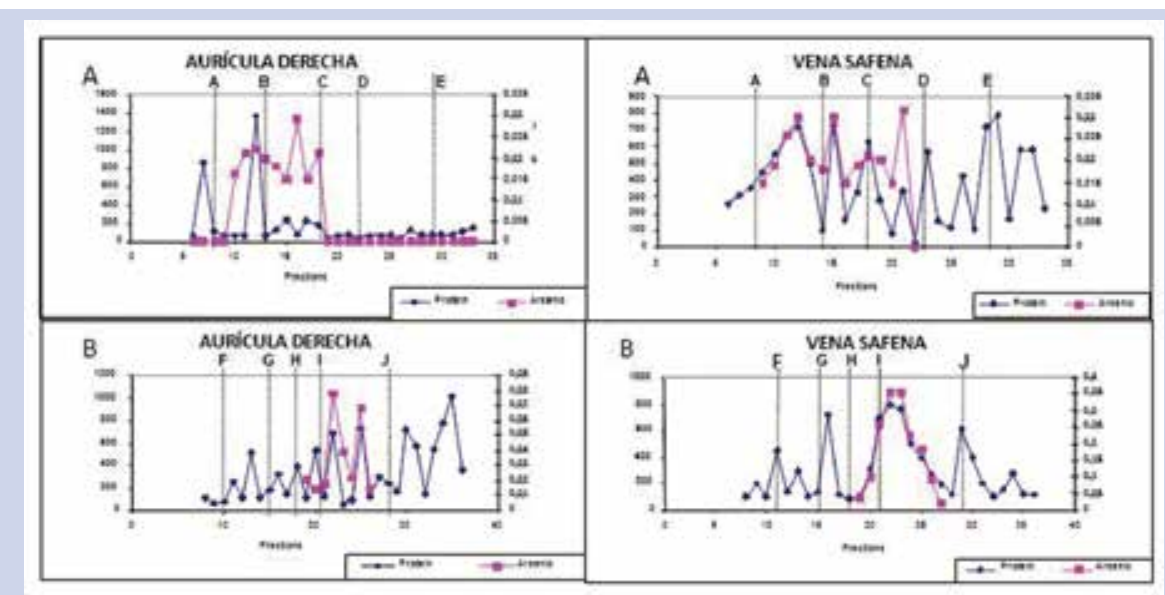

Columna Superdex: A) Aprotinina $6500 \mathrm{Da}$. B) Vitamina B-12 $1576 \mathrm{Da}$

C) Selenocisteina $334 \mathrm{Da} . \mathrm{D})$ Selenometionina $198 \mathrm{Da}$. E) Selenourea $123 \mathrm{Da}$

Columna Phenomenex: F) Sangre de Bovino 69000 Da. G): Anhidrasa carbónica 29000 Da. H) Citocromo C 12400 Da. I) Selenocisteina 334 Da. J) Selenometionina 198 Da 


\section{Figura 3: Cromatogramas de las especies As(III) y As(V) correspondientes a las Fracciones 21-26 del citosol de AUD de un paciente.}
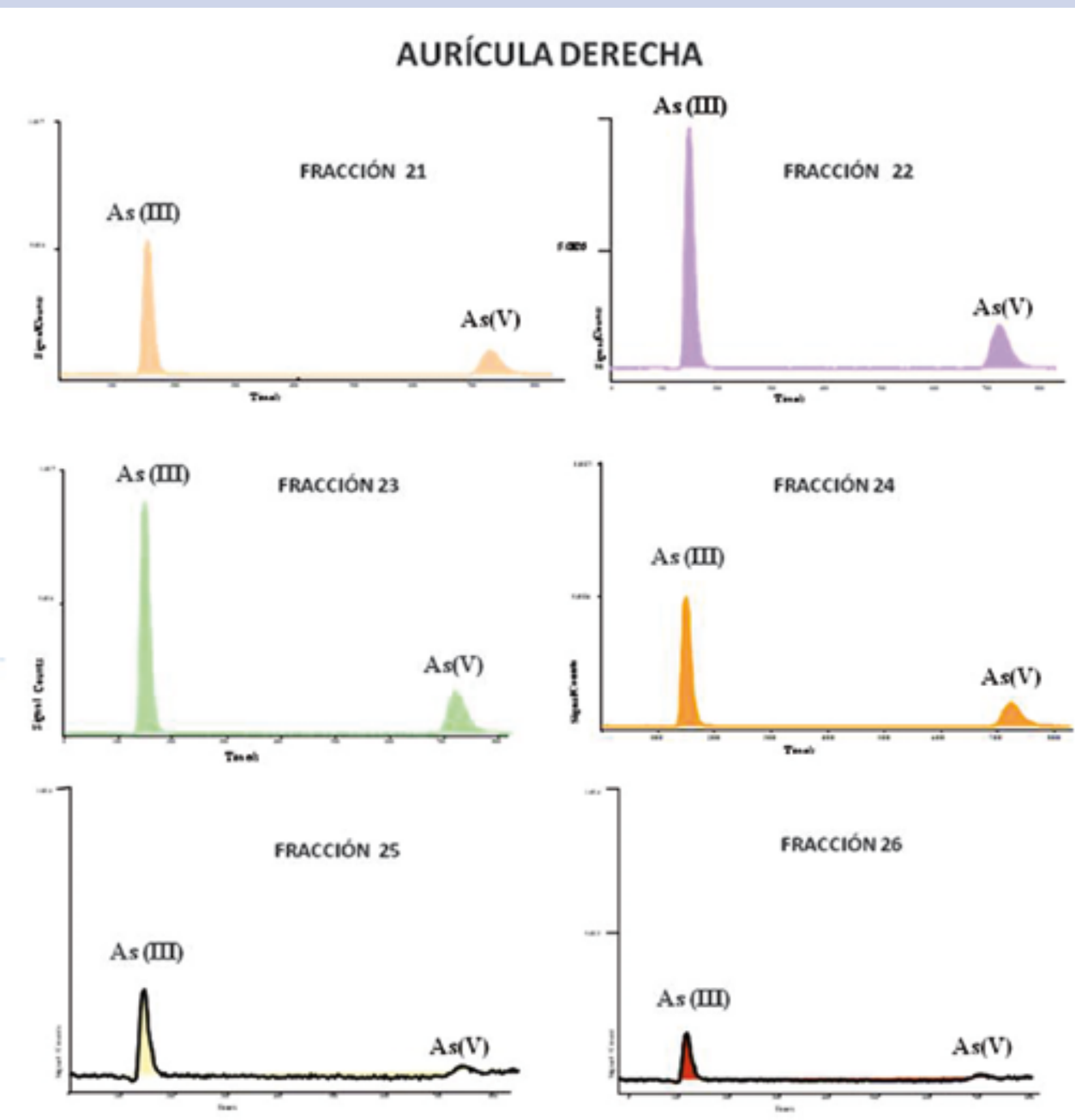

(media 4,9 ugg-1) se encontraron en la AUD .

Unión As-proteína: En la parte A de la Figura 1 se muestran perfiles característicos de la cuantificación del As total $(\mu \mathrm{g})$ y proteína total $(\mu \mathrm{g})$ en conjuntos sucesivos de fracciones separadas en la columna MonoQ HR5/5. Se observa la asociación de los perfiles del As y de las proteínas en fracciones contiguas 21- 26 de la AUD y VS. Más del $50 \%$ del As estaba unido a proteínas de masa superior a $80 \mathrm{kDa}$.

En la parte B de la Figura 1, se muestran los cromatogramas obtenidos en el tándem para la fracción 22 obtenida de la AUD y la fracción 24 obtenida de la VS. La parte $\mathrm{C}$ de la Figura 1 muestra que As y proteína aparecen al mismo tiempo de retención.

La Figura 2A muestra resultados del paciente S-6. El $10 \%$ del As estuvo asociado a péptidos entre 330 y 7.000 Da los que pudieran corresponder a complejos con metalotioneinas o complejos de la glutationa (As(SG)3). En la Figura 2B se observa que el As está unido a proteínas entre 0.3 y $12.4 \mathrm{kDA}$, tanto en VS como AD.

\section{Especiación de As en citosol}

La Figura 3 muestra la presencia de As (III) y As (V) en todas las fracciones (21-26) en AUD de un paciente. La razón As (III)/ As (V) fue constante en la mayoría de las fracciones analizadas. Se encontró una recuperación de [As (III) + As (V)] de 90 - 95\% cuando se determinó As total en cada fracción. 


\section{Discusión}

Este trabajo puede considerarse como un primer paso en la investigación de la bioacumulación de As y de la respuesta bioquímica del tejido CV procedente de individuos expuestos crónicamente a As. Hasta este momento no puede conocerse a qué proteína específica está asociado el As; sin embargo, los resultados ayudan a clarificar algunos aspectos del metabolismo del As en el cuerpo humano.

Aquí reportamos los valores del As total $(\mu \mathrm{g})$ y los perfiles de proteína $(\mu \mathrm{g})$ de los tejidos de AUD y VS en las diferentes fracciones obtenidas después del fraccionamiento por la columna MonoQ HR 5/5, como también la fracción de concentración máxima de la asociación entre As y proteína. Las proteínas totales y As total se distribuyeron en una amplia gama de fracciones y la similitud de los perfiles proteicos y los niveles de concentración de As para ambos tejidos, especialmente para VS, podría reflejar la asociación As - proteína.

Las proteínas unidas al As poseen un peso molecular superior a $80 \mathrm{kDA}$. Las fracciones representan sobre el $50 \%$ del contenido de As y, por lo tanto, la mayor masa molecular de la proteína del citosol después del fraccionamiento está asociado con altos niveles de As. En las fracciones analizadas, el As se une a bio-compuestos de masa molecular superiores a $80 \mathrm{kDA}$ como la transferrina y albúmina. No obstante, debido a la fragmentación celular durante los procesos experimentales, es importante tener presente la capacidad de separación de las proteínas en las columnas de exclusión molecular. Ello, junto a la centrifugación a $30.000 \mathrm{~g}$ para obtener la fracción microsomial, son probablemente las principales causas de la fragmentación y las razones por las cuales As se encuentra asociado a proteínas de diferentes masas moleculares. En este contexto, el As(L16C) 3 podría representar una subunidad de la estructura cuaternaria de la proteína nativa. Los estudios realizados con la columna Phenomenex mostraron que aproximadamente el $25 \%$ del As presente en el tejido pudiera estar unido a proteínas entre 0.3 y $12.4 \mathrm{kDA}$, que pudieran corresponder a moléculas del Citocromo - $\mathrm{C}$.

En investigaciones realizadas por otros autores ${ }^{3}$, se mostró que el As interacciona con la glutationa a través de los grupos sulfhidrilos, formando el complejo As(SG) 3 y con la cisteína para producir $\mathrm{As}(\mathrm{CYS})_{3}$ en las proteínas entre 300 y 8000 kDA, llamadas Arsc gene operon que sintetizan determinados microorganismos para evacuar al
As del citoplasma de sus células. Biomoléculas de peso molecular análogo aparecen en los estudios realizados con la columna Superdex donde el $10 \%$ del As está asociado a péptidos entre 330 y $7000 \mathrm{Da}$, los que pudieran corresponder a complejos con metalotioneinas o complejos de la glutationa $\left(\mathrm{As}(\mathrm{SG})_{3}\right)$.

Nuestros resultados mostraron que sólo As(III) y As(V) están presentes en el caso de la AUD, siendo el As (III) la especie mayoritaria en una relación de 5:1 con respecto al $\mathrm{As}(\mathrm{V})$. Este resultado es interesante, considerando que la única especie presente en las aguas de bebida es el As(V). También es sorprendente que las especies metiladas no estén presentes en la AUD, fundamentalmente considerando que son las especies involucradas en el mecanismo de la detoxificación. Sin embargo, estudios ${ }^{12}$ similares, realizados en el tejido cardíaco de pollos que recibieron As a través del agua de bebida, entregaron resultados comparables a los obtenidos en el presente estudio.

La ausencia de las especies metiladas en la AUD y VS en humanos puede deberse, bien a la ausencia del agente metilante S-Adenosilmetionina, o a que ciertas dosis de As inorgánico puedan interrumpir el mecanismo de la metilación oxidativa. En investigaciones ${ }^{7}$ similares se encontró la incapacidad de las especies metiladas de unirse a las proteínas y otros constituyentes de las células de tejidos cardíacos.

En conclusión, en estos estudios realizados en tejidos de pacientes expuestos a As en la II Región de Antofagasta, que han requerido cirugía de revascularización coronaria, se evidencia la asociación de As (III) a proteínas citosólicas, sugiriendo que el As está unido a bio-compuestos de diferente masa molecular a través de grupos sulfhidrilos vecinales. Es probable que el As en AUD y VS se una a fracciones proteicas de masa molecular superior a $80 \mathrm{kDAy}$ a subunidades de la estructura cuaternaria de la proteína nativa.

\section{Agradecimientos}

Se agradece el financiamiento de esta Investigación a Asistencia Técnica Universidad de Antofagasta - Chile, Departamento de Química Analítica de la Universidad Complutense de Madrid - España. El aporte de los tejidos Cardiovasculares en estudio a Clínica Antofagasta - Chile y Hospital Clínico de la Universidad Católica de Chile. 


\section{Referencias:}

1. SOLAR C, PIZARRO I, ROMAN D. Presencia de altos niveles de arsénico en tejidos cardiovasculares de pacientes de áreas contaminadas de Chile. Revista Chilena de Cardiología. 2012; 30: 221 - 27.

2. FERRECCIO C, SANCHA AM. Arsenic exposure and its impact on health in Chile. J Health Popul Nutr. 2006; 24: $164-75$.

3. FARRER BT, MCCLURE CP, PENNER-HAHN JE, PECORARO V L. Arsenic (III) - cysteine interactions stabilize three-helix bundles in aqueous solution. Inorg Chem. 2000; 39: $5422-23$.

4. MELIKER JR, FRANZBLAU A, SLOTNICK MJ, NRIAGU JO. Major contributors to inorganic arsenic intake in southeastern Michigan. Int. J Hyg Environ - Health. 2006; 209: 399 - 411 .

5. PIZARRO I, GÓMEZ M, CÁMARA C, PALACIOS MA, ROMÁN - SILVA DA. Evaluation of arsenic species protein binding in cardiovascular tissues by bidimensional chromatography with ICP - MS detection. J Anal At Spectrom. 2004; 19: $292-96$.

6. ROY P, SAHA A. Metabolism and toxicity of arsenic: A human carcinogen. Curr Sci. 2002; 82: 38 - 45.

7. JAGER D, JUNGBLUT PR, MULLER-WERDAN U. Separation and identification of human heart proteins. J Chromatogr B. 2002; 771: 131-53.

8. TSENG CH. Metabolism of inorganic arsenic and non cancerous health hazards associated with chronic exposure in humans. J Environ Biol. 2007; 28: 349 - 57.

9. KANG J. Molecular and cellular mechanisms of cardiotoxicity. Environ Health Perspect. 2001; 109: 27 - 34.

10. ROMÁN DA, PIZARRO I, RIVERA L, CÁMARA C, PALACIOS MA, GÓMEZ MM, et al. An approach to the arsenic status in cardiovascular tissues of patients with coronary heart disease. Hum Exp Toxicol. 2010; 30: $1150-64$.

11. CERNA E, OCHOA Y, MENDOZA R, BADI MH, GALLEGOS G, LANDEROS J. Assessment of protein quantification methods in Tetranychus urticae, as a potential tool for resistance detection to pesticides. International Journal of experimental Botany. 2010; 79: 147 - 52 .

12. PIZARRO I, GÓMEZ MM, FODOR P, PALACIOS MA, CÁMARA C. Distribution and Biotransformation of Arsenic species in chicken cardiac and muscle tissues. Biological Trace Element Research. 2004; 99: 129 - 144. 